\title{
A novel mechanism underlies caspase-dependent conversion of the dicer ribonuclease into a deoxyribonuclease during apoptosis
}

Xiao Ge ${ }^{1, *}$, Xiang Zhao ${ }^{1, *}$, Akihisa Nakagawa ${ }^{2, *}$, Xinqi Gong ${ }^{1}$, Riley Robert Skeen-Gaar ${ }^{2}$, Yong Shi $^{2}$, Haipeng Gong ${ }^{1}$, Xinquan Wang ${ }^{1}$, Ding Xue ${ }^{1,2}$

${ }^{I}$ School of Life Sciences, Tsinghua University, Beijing 100084, China; ${ }^{2}$ Department of Molecular, Cellular and Developmental Biology, University of Colorado, Boulder, CO 80309, USA

During $C$. elegans apoptosis, the dicer ribonuclease (DCR-1) is cleaved by the cell death protease CED-3 to generate a truncated DCR-1 (tDCR-1) with one and a half ribonuclease III (RNase III) domains, converting it into a deoxyribonuclease (DNase) that initiates apoptotic chromosome fragmentation. We performed biochemical and functional analyses to understand this unexpected RNase to DNase conversion. In full-length DCR-1, tDCR-1 DNase activity is suppressed by its N-terminal DCR-1 sequence. However, not all the sequence elements in the N-terminal DCR-1 are required for this suppression. Our deletion analysis reveals that a 20-residue $\alpha$-helix sequence in DCR-1 appears to define a critical break point for the sequence required for suppressing tDCR-1 DNase activity through a structure-dependent mechanism. Removal of the N-terminal DCR-1 sequence from tDCR-1 activates a DNA-binding activity that also requires the one half RNase IIIa domain, and enables tDCR-1 to process DNA. Consistently, structural modeling of DCR-1 and tDCR-1 suggests that cleavage of DCR-1 by CED-3 may cause a conformational change that allows tDCR-1 to bind and process DNA, and may remove steric hindrance that blocks DNA access to tDCR-1. Moreover, a new DNase can be engineered using different RNase III domains, including the one from bacterial RNase III. Our results indicate that very distantly related RNase III enzymes have the potential to cleave DNA when processed proteolytically or paired with an appropriate partner that facilitates binding to DNA. We suggest the possibility that this phenomenon may be extrapolated to other ribonucleases.

Keywords: C. elegans; dicer ribonuclease; deoxyribonuclease; caspase; apoptosis; conversion

Cell Research (2014) 24:218-232. doi:10.1038/cr.2013.160; published online 10 December 2013

\section{Introduction}

Dicer is a member of the double-stranded RNA (dsRNA)-specific ribonuclease III (RNase III) family that processes dsRNA substrates to generate small RNAs such as miRNAs and siRNAs, which are important for various gene silencing events [1-8]. This protein family is not known to possess deoxyribonuclease (DNase) activity $[9,10]$ until the recent finding that the $C$. elegans

\footnotetext{
*These three authors contributed equally to this work.

Correspondence: Ding Xue

Tel: +1-303-492-0271

E-mail: Ding.Xue@Colorado.EDU

Received 23 August 2013; revised 15 October 2013; accepted 16 October 2013; published online 10 December 2013
}

dicer ribonuclease (DCR-1) can be converted into a DNase upon caspase cleavage during apoptosis [11]. During C. elegans apoptosis, DCR-1 is cleaved by the cell death protease CED-3 to generate a C-terminal cleavage product (tDCR-1), which acquires a DNase activity to initiate apoptotic chromosome fragmentation. Cell death analysis in $C$. elegans reveals that inactivation of $d c r-1$ reduces embryonic cell death and blocks chromosomal DNA fragmentation during apoptosis [11]. Importantly, both cell death defects can be fully rescued by the expression of tDCR-1 under the control of the endogenous $d c r-1$ gene promoter $\left(\mathrm{P}_{d c r-1} \mathrm{tDCR}-1\right)$, but not by the expression of a DCR-1 mutant $\left(\mathrm{P}_{d c r-1} \mathrm{DCR}-1(\mathrm{D} 1472 \mathrm{E})\right)$ that is resistant to cleavage by CED-3, indicating that generation of tDCR-1 is both necessary and sufficient for normal apoptosis in C. elegans [11]. 
Dicer ribonucleases are highly conserved. Most of them contain an N-terminal helicase domain, a PAZ (Piwi, Argo, and Zwille) domain in the middle, and two tandem RNase III domains followed by a dsRNA-binding domain at the C-terminus (Figure 1A) $[9,10]$. Biochemical and structural studies of the Dicer ribonucleases indicate that the two RNase III domains form a single processing center by folding into an intramolecular dimer, with each domain contributing to the hydrolysis of one strand of the RNA duplex substrate through four highly conserved acidic residues that coordinate two catalytic $\mathrm{Mg}^{2+}$ cations $[12,13]$. Interestingly, CED-3 cleaves $C$. elegans DCR-1 in the middle of its first RNase III domain, yielding a Cterminal cleavage product, tDCR-1, with one and a half RNase III domains and a dsRNA-binding domain (Figure 1A). This proteolytic processing disables the dsRNA dicing activity of DCR-1, but activates a DNase activity that nicks chromatin DNA to generate 3' OH DNA ends through the same conserved acidic residues and catalytic $\mathrm{Mg}^{2+}$ [11]. The nicked chromosomal DNA is further processed by downstream apoptotic nucleases, including CPS-6 and CRN-1, leading to generation of doublestranded DNA breaks $[14,15]$. The mechanism underlying this unexpected RNase to DNase conversion is not understood.

In this study, we perform biochemical and functional analyses to investigate how DCR-1 RNase is proteolytically converted into a DNase to promote apoptosis. We find that not all the sequence elements in the N-terminal DCR-1 are required to inhibit tDCR-1 DNase. Instead, only a certain length of the N-terminal DCR-1 sequence is needed to suppress tDCR-1 DNase activity. In particular, from our deletion analysis, we identify a 20-residue $\alpha$-helix in DCR-1 that defines a critical break point for the sequence required for inhibiting this DNase activity and that can be replaced by an unrelated $\alpha$-helix of the same length, but not by a 20 -residue unstructured sequence. In addition, unrelated proteins, such as green fluorescent protein (GFP) and glutathione S-transferase (GST), can potently suppress tDCR-1 DNase activity when fused to the N-terminus of tDCR-1. These findings indicate that the basis of tDCR-1 DNase suppression is likely through structural interference rather than through a sequence-specific mechanism. We also show that tDCR-1 acquires DNA-binding ability upon removal of its N-terminal inhibitory sequence, which correlates with its ability to process DNA. Consistently, structural modeling of DCR-1 and tDCR-1 suggests that CED-3 cleavage of DCR-1 may remove potential steric hindrance and cause a conformational change, allowing tDCR-1 to bind and process DNA. Moreover, new DNases can be generated by pairing a different RNase III domain, including the one from bacterial RNase III, with a different onehalf RNase III domain. Our study thus provides important insight into how an RNase can be converted into a DNase to drastically affect important cellular events.

\section{Results}

Most DCR-1 sequences $N$-terminal to tDCR-1 are dispensable for inhibition of the tDCR-1 DNase activity

As we reported previously [11], cleavage of DCR-1 by CED-3 activates a DNase activity that can nick and linearize supercoiled (SC) plasmid DNA to generate $3^{\prime}$ OH DNA ends (Figure 1B, lanes 1-4). As full-length DCR-1 does not cleave or nick DNA (Figure 1B, lane 3), tDCR-1 DNase must be suppressed by the N-terminal sequence of DCR-1 (residues 1-1472) and activated upon CED-3 cleavage (Figure 1B, lane 4). To probe the region in DCR-1(1-1472) responsible for inhibiting tDCR-1 DNase, we made a series of DCR-1 N-terminal deletions that progressively remove different domains in the DCR1(1-1472) sequence (Figure 1A). These DCR-1 mutants were synthesized as $\mathrm{C}$-terminal fusions to GST in rabbit reticulocyte lysate, affinity purified using Glutathione Sepharose, and then digested with PreScission Protease (PPase) to remove the GST tag. Purified DCR-1 proteins were incubated with supercoiled plasmid DNA in the presence or absence of CED-3, and assessed for the DNase activity using the plasmid DNA nicking assay and the in vitro TUNEL (Terminal Deoxynucleotidyl Transferase (TdT)-mediated dUTP nick end labeling) assay (Figure 1B) [11]. In parallel, we expressed these DCR-1 mutants under the control of the endogenous $d c r-1$ promoter and examined whether they could rescue the cell death defect of $d c r-1$ (ok247) animals, which is caused by the lack of tDCR-1 DNase [11].

We found that progressive removal of the helicase domain (DCR-1(740-1845)), the PAZ domain (DCR-1(12971845)), and a small portion of the RNase IIIa domain (DCR-1(1345-1845)) did not affect CED-3 cleavage of DCR-1 (Supplementary information, Figure S1A, lanes 1-2 and 5-10) and CED-3-dependent activation of DCR-1 DNase in vitro (Figure 1B, lanes 3-12). In vivo, these truncated proteins fully rescued the cell death defect of $d c r-1$ ( ok247) animals, when expressed from the $d c r-1$ promoter (Figure 1C-1E). To evaluate whether CED-3 cleavage at Asp1472 of these DCR-1 truncations is critical for activating DCR1 DNase and their proapoptotic functions in vivo (Figure 1A), we generated Asp1472 to Glu substitution (D1472E) in these DCR-1 mutants [11], which blocked CED-3-mediated production of tDCR-1 (Supplementary information, Figure S1B, lanes 1-8). These D1472E DCR-1 mutants could not be activated into a DNase by CED-3 (Supple- 
A

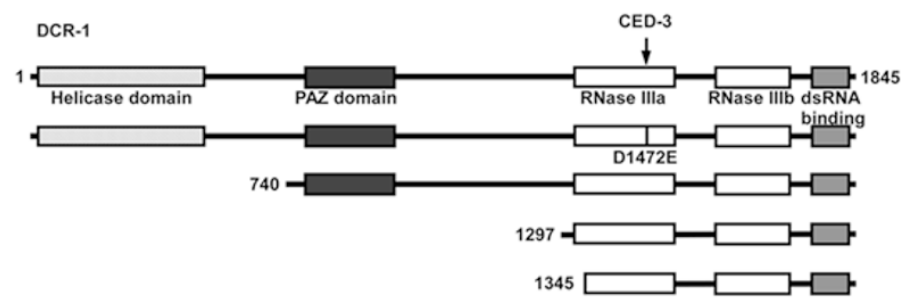

C

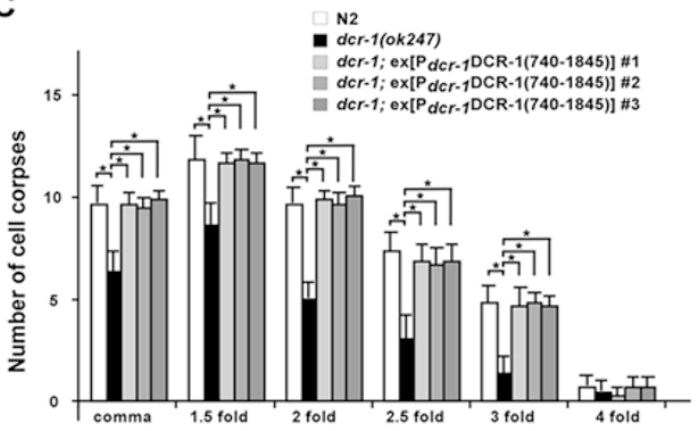

B
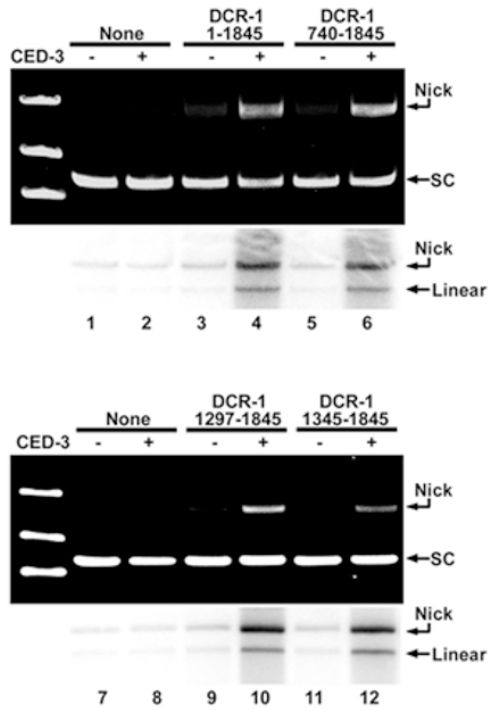

D

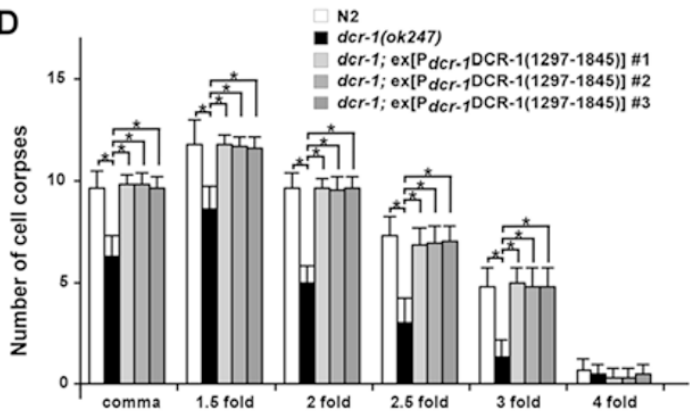

$\mathbf{F}$

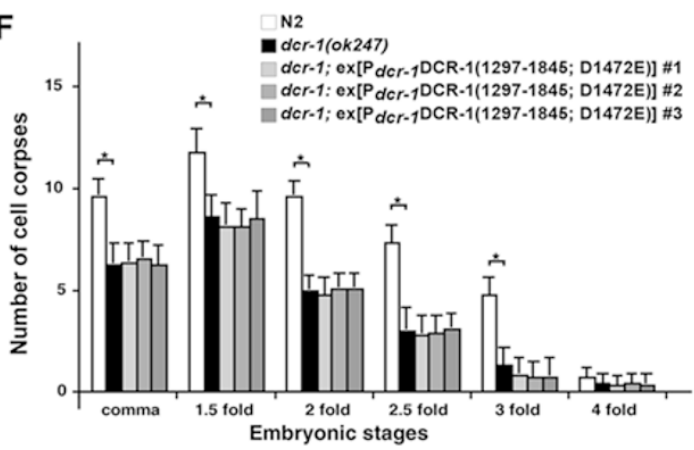

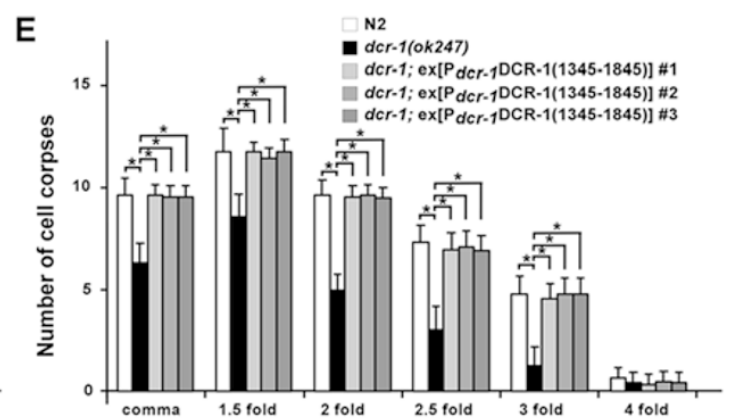

G

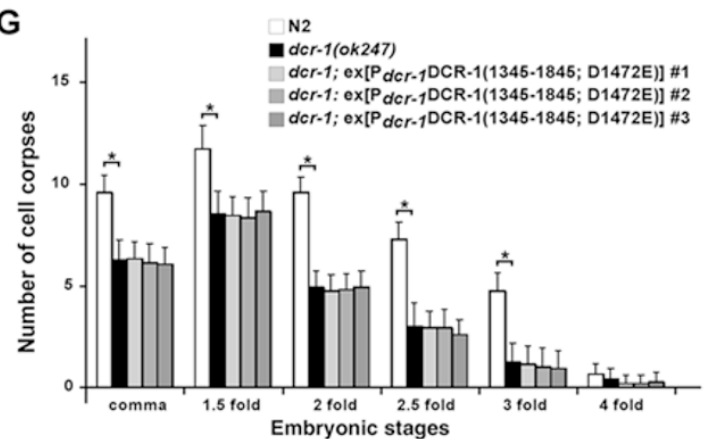

Figure 1 Role of DCR-1 N-terminal domains in suppression of tDCR-1 DNase activity. (A) A schematic diagram of DCR-1 deletions. The arrow indicates CED-3 cleavage site at Asp1472. (B) Inhibition of tDCR-1 DNase by the sequences from DCR1(1-1472), DCR-1(740-1472), DCR-1(1297-1472), and DCR-1(1345-1472). Purified DCR-1 proteins were incubated with supercoiled pPD49.78 plasmid (200 ng) in the absence or presence of purified CED-3 for $1 \mathrm{~h}$ at $37^{\circ} \mathrm{C}$. One half of the sample was resolved on $1 \%$ agarose gel and then stained with GelRed (top). The other half was labeled with $\left[\alpha-{ }^{32} \mathrm{P}\right] \mathrm{dCTP}$ using TdTase for $2 \mathrm{~h}$ at $37{ }^{\circ} \mathrm{C}$, resolved on $1 \%$ agarose gel, fixed and dried for autoradiography (bottom). (C-G) Rescue of the cell death defect of $d c r-1$ (ok247) animals by expression of DCR-1 truncations. Cell corpses were scored in N2, dcr-1(ok247), and dcr-

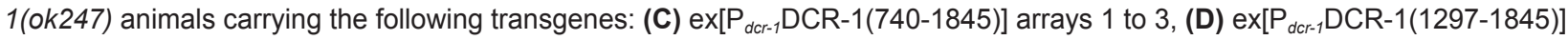

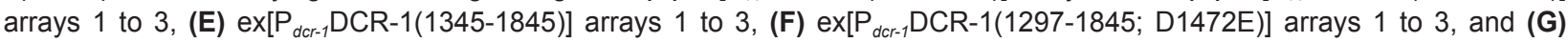
ex[ $P_{d c r-1}$ DCR-1(1345-1845; D1472E)] arrays 1 to 3 . The $x$ axis represents different embryonic stages, $y$ axis represents average number of cell corpses scored, and error bars represent standard deviations (SD). ${ }^{*} P<0.001$. All other points had $P>0.05$. 
mentary information, Figure S2, lanes 7-10) and failed to rescue the cell death defect of $d c r-1$ (ok247) animals (Figure $1 \mathrm{~F}$ and $1 \mathrm{G}$ ). These results indicate that the DCR1(1345-1472) sequence is sufficient to suppress tDCR-1 DNase activity and that the N-terminal helicase domain, the PAZ domain, and a part of the RNase IIIa domain (1297-1344) are dispensable for this suppression.

A 20-residue $\alpha$-helix in the RNase IIIa domain defines a break point for the DCR-1 sequence required to suppress the tDCR-1 DNase activity

Interestingly, two further deletions in the RNase IIIa domain, DCR-1(1405-1845) and DCR-1(1420-1845), caused activation of DNase in the absence of CED-3 (Figure 2A and 2B, lanes 5-8), whereas DCR-1(1385$1845)$ with a smaller deletion remained dependent on CED-3 to activate its DNase activity (Figure 2B, lanes 3 and 4). These results indicate that the DCR-1(14051472) region is incapable of suppressing tDCR-1 DNase activity. Consistent with this in vitro observation, both DCR-1(1405-1845) and DCR-1(1420-1845) as well as their CED-3-resistent forms (Supplementary information, Figure S1B, lanes 9-16), DCR-1(1405-1845; D1472E) and DCR-1(1420-1845; D1472E), fully rescued the cell death defect of $d c r-1(o k 247)$ animals (Figure 2C-2F). By contrast, only DCR-1(1385-1845), but not DCR-1(13851845; D1472E), could rescue the cell death defect of dcr-1(ok247) animals (Figure $2 \mathrm{G}$ and $2 \mathrm{H}$ ). These results indicate that DCR-1(1405-1845) and DCR-1(1420-1845) can promote apoptosis independent of CED-3.

As the DCR-1(1385-1472) sequence can suppress tDCR-1 DNase activity but the DCR-1(1405-1472) sequence cannot, the 20-residue DCR-1(1385-1404) sequence appears to define a critical break point for inhibiting tDCR-1 DNase activity. To understand how the DCR-1(1385-1404) sequence can critically contribute to the suppression of tDCR-1 DNase activity, we performed secondary structure prediction of this sequence [16] and found that it likely forms an $\alpha$-helix. This relatively rigid $\alpha$-helical structure in combination with the structure formed by DCR-1(1405-1472) may severely interfere with the proper function of tDCR-1 DNase. To verify this possibility, we replaced residues 1385-1404 of the DCR-1(1385-1845) protein with an unrelated $\alpha$-helix of the same length [helix-DCR-1(1405-1845)] or an unstructured GGGS-repeat of the same length [GS-DCR-1(1405-1845)](Figure 3A). Both helixDCR-1(1405-1845) and GS-DCR-1(1405-1845) were cleaved normally by CED-3 as DCR-1(1385-1845) (Supplementary information, Figure S1C). However, only helix-DCR-1(1405-1845) showed CED-3-dependent activation of tDCR-1 DNase (Figure 3B, lanes 3 and 4).
By contrast, GS-DCR-1(1405-1845) displayed a CED3 -independent DNase activity similar to DCR-1(14051845) or DCR-1(1420-1845) (Figure 2B and Figure 3B, lanes 5 and 6). In vivo, helix-DCR-1(1405-1845), but not helix-DCR-1(1405-1845; D1472E), rescued the cell death defect of $d c r-1$ (ok247) animals (Figure 3C and 3D), confirming that helix-DCR-1(1405-1845) needs to be activated by CED-3 to promote apoptosis. In contrast, both GS-DCR-1(1405-1845) and GS-DCR-1(1405-1845; D1472E) fully rescued the cell death defect of $\mathrm{dcr}$ 1(ok247) animals (Figure 3E and 3F), displaying CED3 -independent proapoptotic activity. These results indicate that the secondary structure of the 20 residues before DCR-1(1405-1472) directly affects the ability of DCR-1(1385-1472) to suppress tDCR-1 DNase activity, and thus, can be regarded as the "critical break point" for suppression under this context.

We then examined whether this 20-residue sequence is also critical for suppressing the DNase activity in fulllength DCR-1. When the DCR-1(1385-1404) sequence was deleted or replaced by an unstructured GGGS-repeat of the same length in full-length DCR-1, the resulting proteins, DCR-1(1-1845; $\Delta 1385-1404)$ and DCR-1(11384-GS-1405-1845), still exhibited CED-3-dependent DNase activity (Supplementary information, Figures S1D and S3). These observations and the above finding that an unrelated $\alpha$-helix of the same length can replace the DCR-1(1385-1404) sequence in DCR-1(1385-1845) to mediate suppression of tDCR-1 suggest that this structure-related suppression of tDCR-1 DNase activity by DCR-1(1385-1404) may not be sequence-specific and that the DCR-1(1385-1404) sequence may be replaced by other N-terminal DCR-1 sequences or even unrelated protein sequences that have a similar structure (see below).

Unrelated protein sequences can also suppress tDCR-1 DNase activity when fused to its $N$-terminus

Indeed, when we fused green fluorescent protein (GFP), an unrelated protein with a large rigid structure, to the N-terminus of tDCR-1 (Figure 4A), the resulting GFP-tDCR-1 fusion, GFP-DCR-1(1473-1845), did not show any DNase activity with or without CED-3 (Figure 4B, lanes 5 and 6) and failed to rescue the cell death defect of $d c r-1$ (ok247) animals when expressed in C. elegans (Figure 4C). These results confirm that an unrelated protein sequence can replace the DCR-1 N-terminal sequence to block tDCR-1 DNase activity both in vitro and in vivo. When the CED-3 cleavage site (STTD) in DCR1 was inserted between GFP and tDCR-1, the resulting GFP-DCR-1(1469-1845) fusion could be cleaved by CED-3 (Supplementary information, Figure S1E), lead- 
A

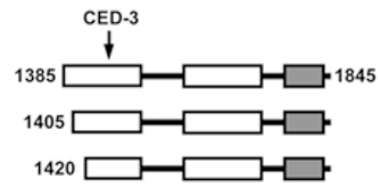

C

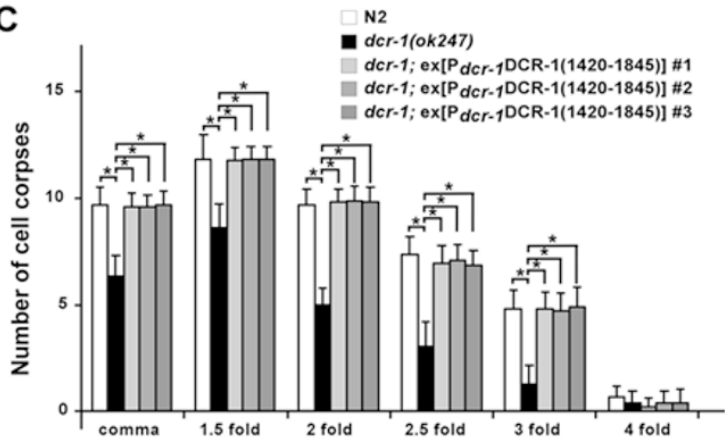

E

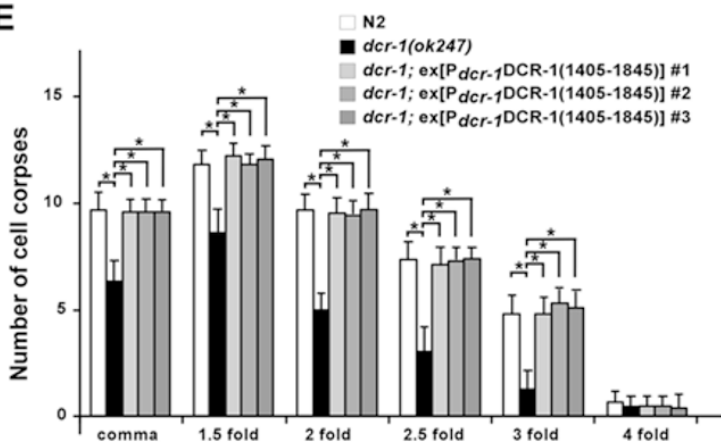

G

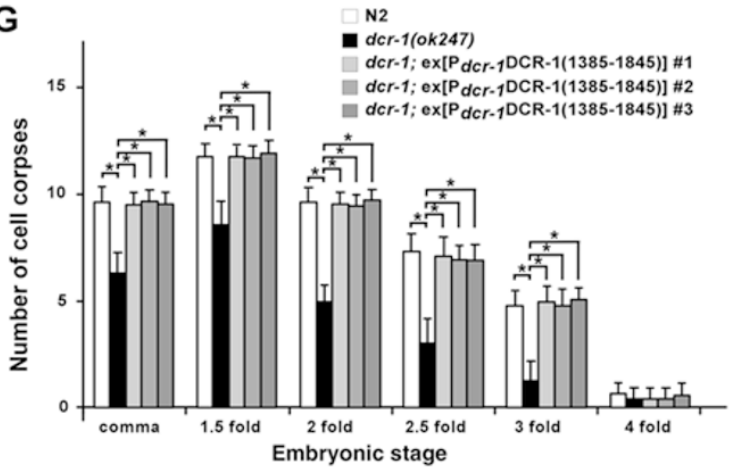

B

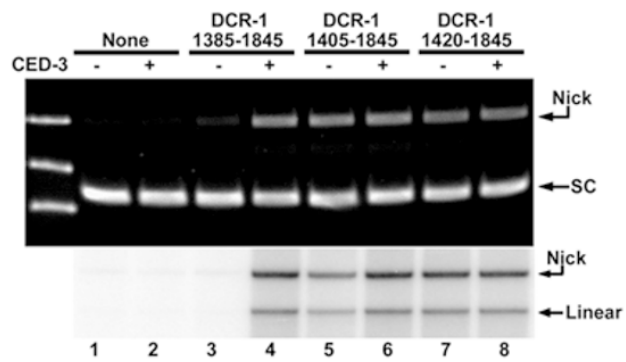

D $\square$ N2

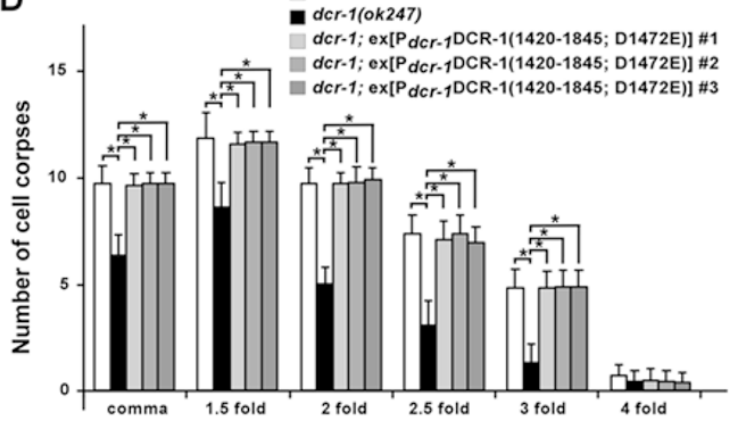

F

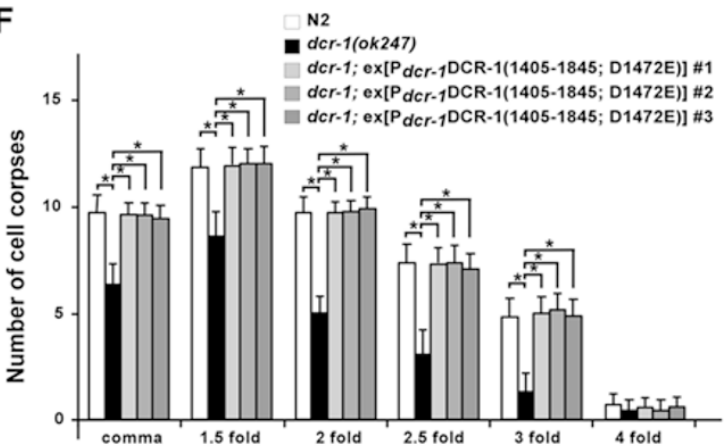

$\mathrm{H}$

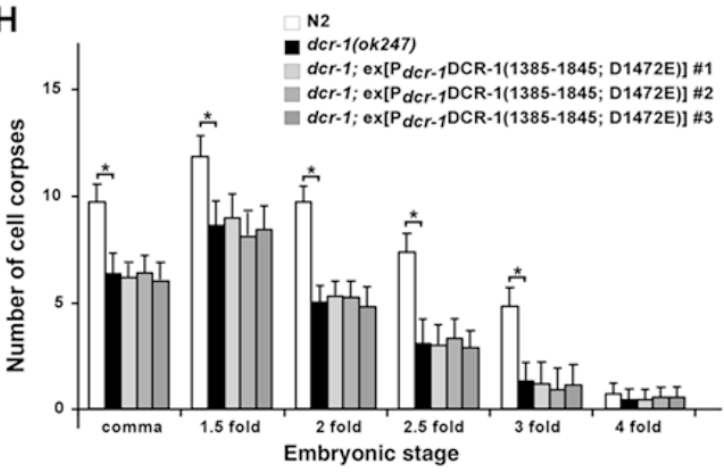

Figure 2 Residues 1385-1404 of DCR-1 define a break point for suppressing tDCR-1 DNase activity. (A) A schematic diagram of DCR-1 deletions. The arrow indicates CED-3 cleavage site at Asp1472. (B) Inhibition of tDCR-1 DNase by the sequence from DCR-1(1385-1472), but not by the sequence from DCR-1(1405-1472) or DCR-1(1420-1472). (C-H) In vivo rescue of the cell death defect of $d c r-1$ (ok247) animals by expression of DCR-1 truncations. Cell corpses were scored in N2, $d c r-1$ (ok247), and dcr-1(ok247) animals carrying the following transgenes: (C) ex[ $P_{d c r-1} \mathrm{DCR}-1$ (1420-1845)] arrays 1 to 3, (D) ex[P $P_{d c r-1} D C R-1\left(1420-1845 ;\right.$ D1472E)] arrays 1 to 3, (E) ex[P $\left.P_{d c r-1} D C R-1(1405-1845)\right]$ arrays 1 to 3, (F) ex[ $P_{d c r-1} D C R-1(1405-1845$;

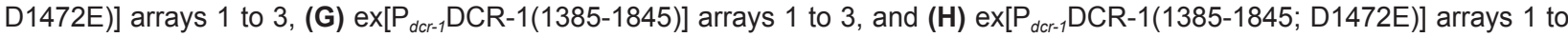
3. The $x$ axis represents different embryonic stages, $y$ axis represents average number of cell corpses scored and error bars represent standard deviations (SD). ${ }^{*} P<0.001$. All other points had $P>0.05$. 
A

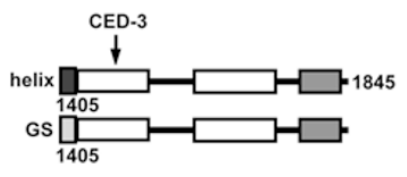

C

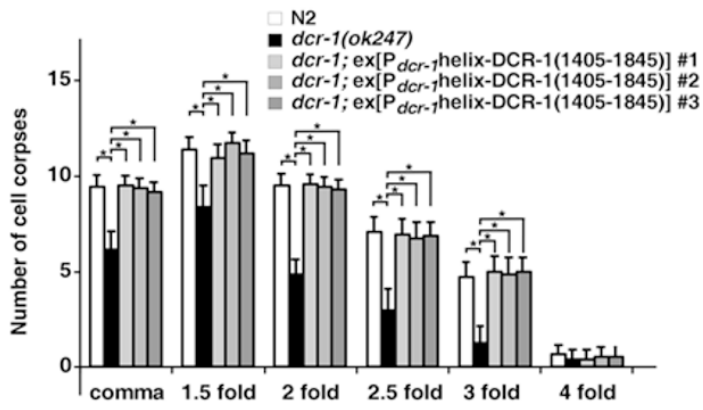

E

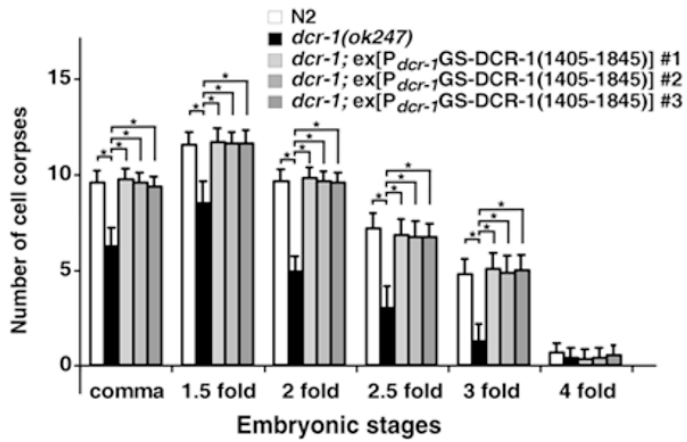

B

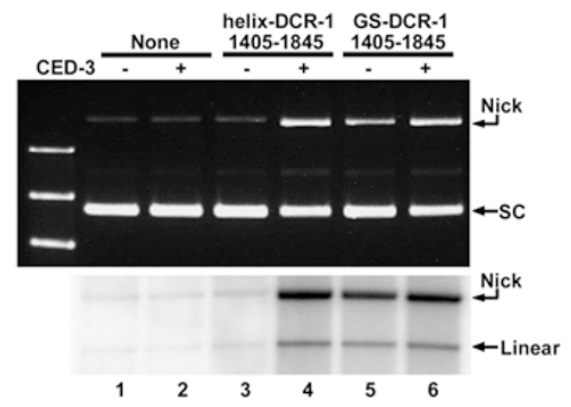

D

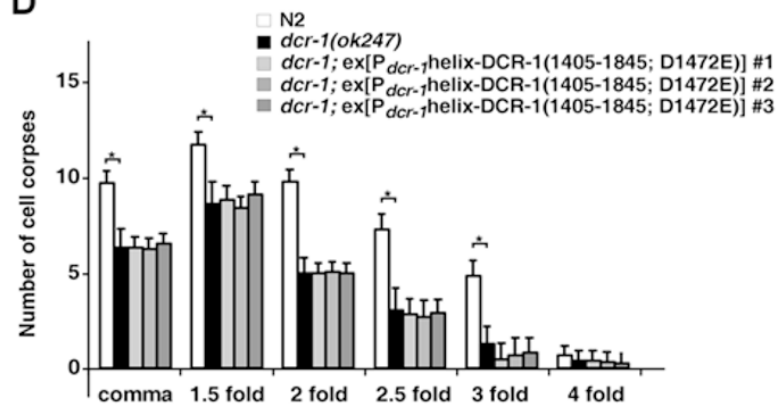

$\mathbf{F}$

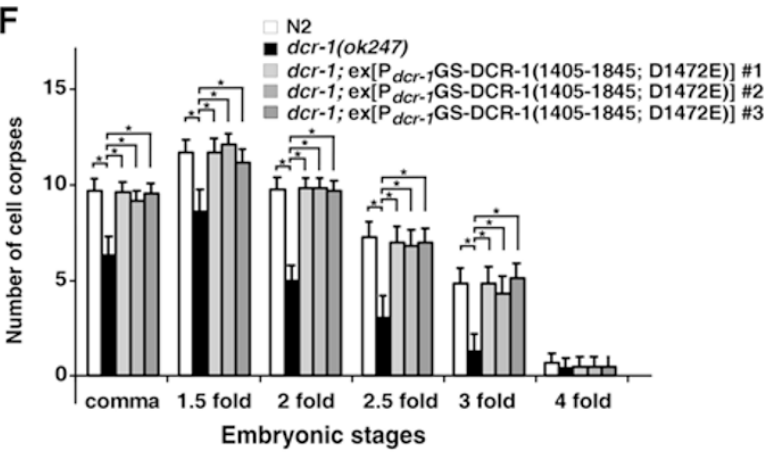

Figure 3 Residues $1385-1404$ of DCR-1 replaced by an unrelated $\alpha$-helix of the same length can suppress tDCR-1 DNase activity. (A) A schematic diagram of DCR-1 replacements generated. The arrow indicates CED-3 cleavage site at Asp1472. Helix and GS represent sequences replacing DCR-1(1385-1404) (see Materials and Methods). (B) Residues 1385-1404 of DCR-1 replaced by an unrelated $\alpha$-helix of the same length, but not by an unstructured sequence of the same length with GGGS repeats, can suppress tDCR-1 DNase activity. (C-F) Rescue of the cell death defect of dcr-1(ok247) animals by expression of DCR-1 mutants. Cell corpses were scored in N2, dcr-1(ok247), and dcr-1(ok247) animals carrying the following

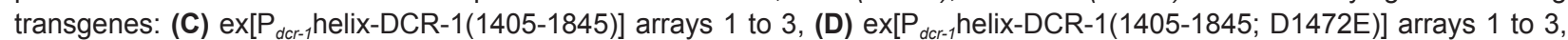

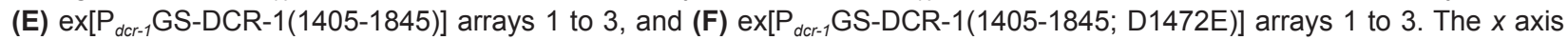
represents different embryonic stages, $y$ axis represents average number of cell corpses scored and error bars represent standard deviations (SD). ${ }^{*} P<0.001$. All other points had $P>0.05$.

ing to CED-3-dependent activation of DNase (Figure 4B, lanes 1-4). In vivo, GFP-DCR-1(1469-1845) fully rescued the cell death defect of $d c r-1$ (ok247) animals (Figure 4D). Fusing GST to tDCR-1 through a PPase cleavage site (PP), GST-PP-DCR-1(1473-1845), also inhibited tDCR-1 DNase (Figure 4B, lane 9), which could be activated upon removal of GST by PPase cleavage (Figure $4 \mathrm{~B}$, lane 10). These results indicate that placing an unrelated protein sequence to the N-terminus of tDCR-1 can also inhibit tDCR-1 DNase activity, probably through a sequence-independent, but structure-dependent mechanism.

The one half RNase IIIa domain is critical for tDCR-1 DNase activity

Dicer ribonucleases cleave dsRNA through their two RNase III domains, each of which is responsible for hydrolysis of one RNA strand via four highly conserved 
A

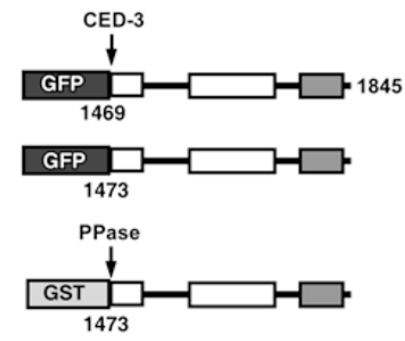

C

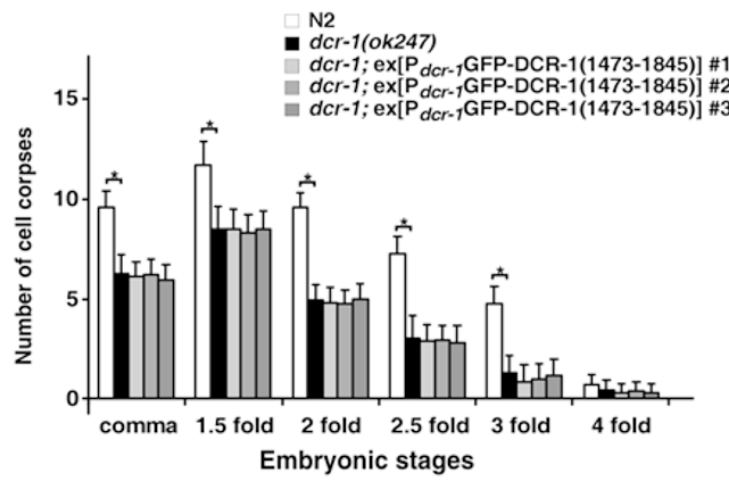

B

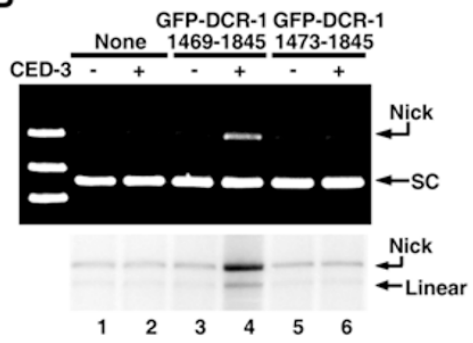

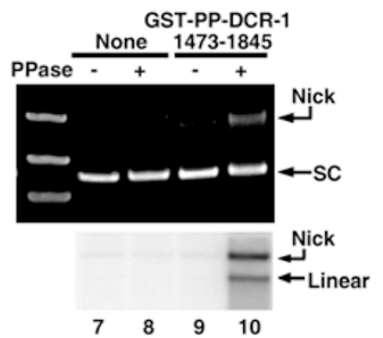

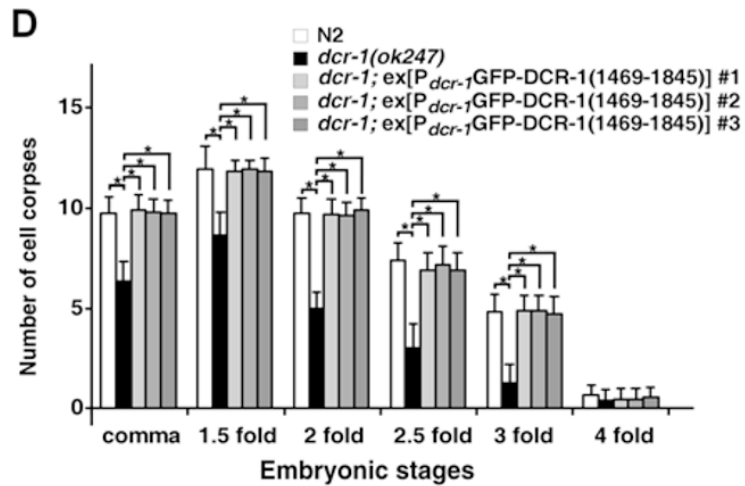

Figure 4 Suppression of tDCR-1 DNase activity by unrelated protein sequences. (A) A schematic diagram of tDCR-1 Nterminally fused with GFP or GST. Arrows indicate CED-3 cleavage site at Asp1472 (upper) or PPase cleavage site right before tDCR-1 (lower). (B) Inhibition of tDCR-1 DNase by N-terminally attached GFP or GST. (C, D) Rescue of the cell death defect of $d c r-1$ (ok247) animals by two different GFP-DCR-1 fusions. Cell corpses were scored in N2, dcr-1(ok247), and dcr1(ok247) animals carrying the following transgenes: (C) ex[P $P_{d c r-1}$ GFP-DCR-1(1473-1845)] arrays 1 to 3 , (D) ex[P dcr-1 $_{1}$ GFPDCR-1(1469-1845)] arrays 1 to 3 . The $x$ axis represents different embryonic stages, $y$ axis represents average number of cell corpses scored and error bars represent standard deviations (SD). ${ }^{*} P<0.001$. All other points had $P>0.05$.

acidic residues that coordinate two $\mathrm{Mg}^{2+}$ cations $[12,13]$. tDCR-1 appears to use the same two-metal-ion catalytic mechanism to hydrolyze DNA [17], because mutations in three of the four catalytic acidic residues in the RNase IIIb domain abolish the DNase activity of tDCR-1 and the dsRNA dicing activity of full-length DCR-1 [11]. We examined whether the remaining one half RNase IIIa domain in tDCR-1, which still contains two catalytic residues (Asp1510 and Glu1513), is important for tDCR-1 to process DNA. We found that deletion of the entire RNase IIIa domain, DCR-1(1560-1845), abolished tDCR-1 DNase activity (Figure 5A and 5B, lanes 5 and 6 , and Supplementary information, Figure S1F, lane 2). Similarly, D1510A/E1513A double mutations rendered DCR-1 incapable of making active tDCR-1 DNase (Figure 5A, 5C and Supplementary information, Figure S1A, lanes 3 and 4). In vivo, both DCR-1(1560-1845) and DCR-1(D1510A/E1513A) failed to promote apoptosis (Figure 5D and 5E). These results indicate that the one half RNase IIIa domain is as important as the complete RNase IIIb domain in making a functional tDCR-1 DN- ase.

DCR-1 binds DNA once cleaved and activated by CED-3

As DCR-1(1473-1845) and DCR-1(1420-1845), but not other N-terminally extended tDCR-1 versions such as DCR-1(1345-1845), can cleave plasmid DNA on their own, we tested whether the DNase activity of these proteins correlates with their ability to bind DNA through UV cross-linking experiments. We expressed GST-DCR-1(1297-1845)-FLAG, GST-DCR-1(13451845)-FLAG, GST-DCR-1(1420-1845)-FLAG, and GST-DCR-1(1297-1845, D1510A/E1513A)-FLAG in Sf-9 insect cells, purified these proteins using Glutathione Sepharose resins, and removed their GST tags using PPase. The purified DCR-1 proteins were then incubated with supercoiled plasmid DNA in the absence or presence of CED-3, irradiated with UV, and pulled down using anti-FLAG resins. The presence of bound DNA was visualized by linearizing the plasmid with BamHI and biotin labeling.

We found that the ability of DCR-1 proteins to bind 
A

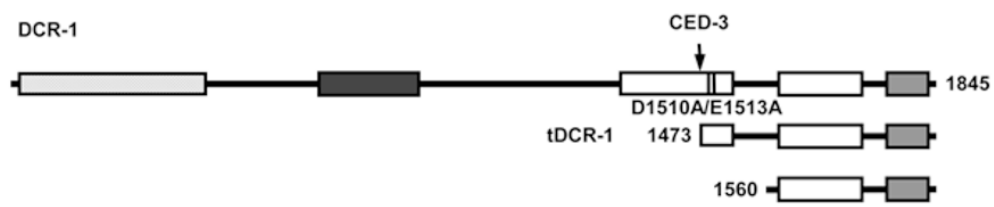

B

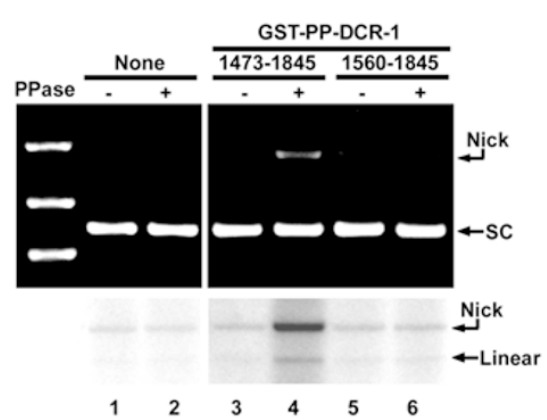

C
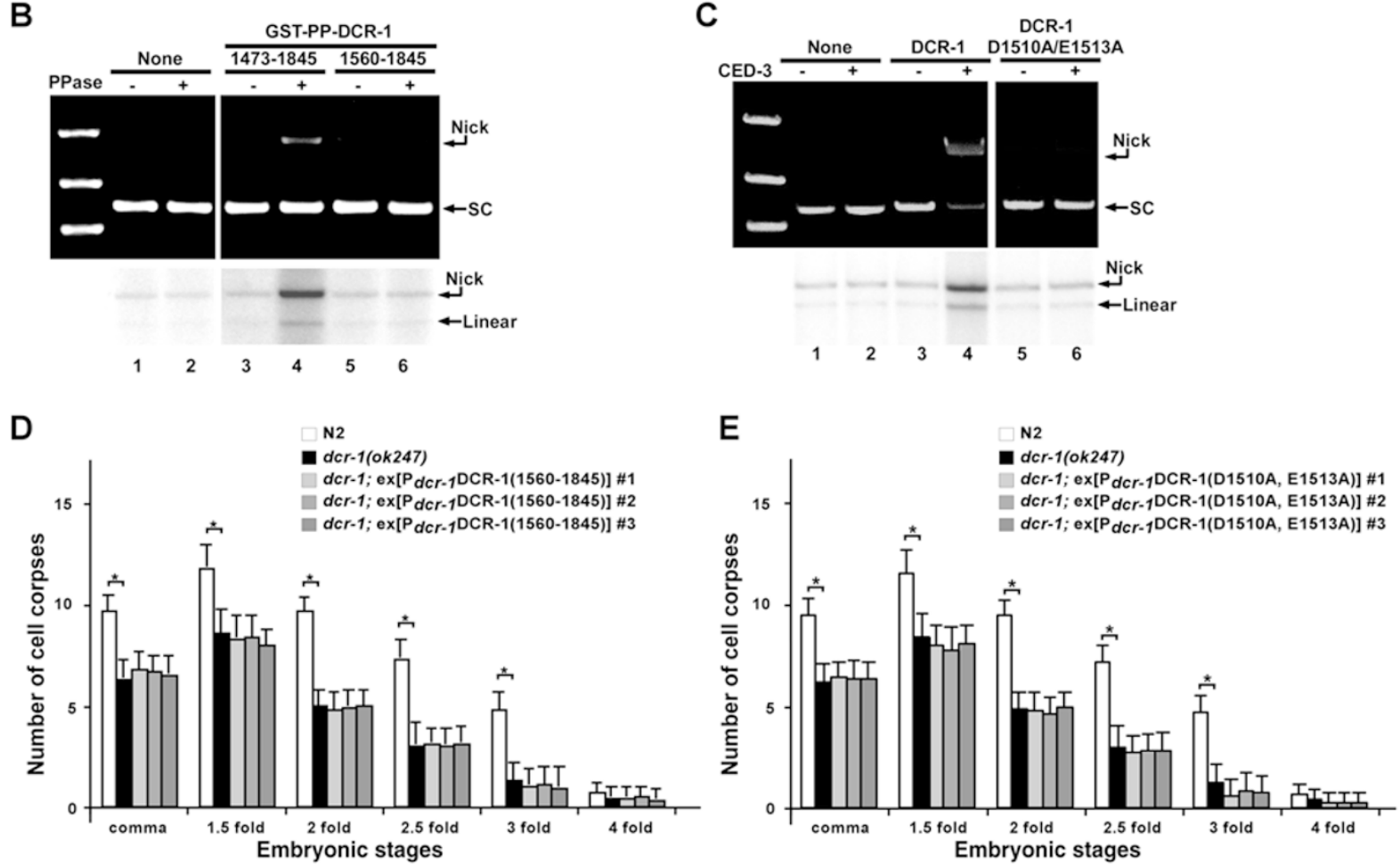

Figure 5 The importance of the one half RNase Illa domain to tDCR-1 DNase. (A) A schematic diagram of DCR-1 mutants. The arrow indicates CED-3 cleavage site at Asp1472. (B) Removal of the remaining RNase IIla domain in tDCR-1 abolishes its DNase activity. (C) Substitutions of the two catalytic residues in the RNase Illa domain of DCR-1 abolish CED-3-dependent DNase activity. (D, E) Rescue of the cell death defect of $d c r-1$ (ok247) animals by the two DCR-1 mutants. Cell corpses were scored in N2, dcr-1(ok247), and dcr-1(ok247) animals carrying the following transgenes: (D) ex[ $\left.P_{d c r-1} D C R-1(1560-1845)\right]$ arrays 1 to 3 , (E) ex[ $\left.P_{d c r-1} D C R-1(D 1510 A, E 1513 A)\right]$ arrays 1 to 3 . The $x$ axis represents different embryonic stages, $y$ axis represents average number of cell corpses scored and error bars represent standard deviations (SD). ${ }^{*} P<0.001$. All other points had $P>0.05$.

DNA strictly correlated with their DNase activity. DCR1(1297-1845) and DCR-1(1345-1845) barely bound DNA in the absence of CED-3 (Figure 6A, lanes 3 and $5)$, but showed greatly enhanced DNA-binding ability in the presence of CED-3 (Figure 6A, lanes 4 and 6). By contrast, DCR-1(1420-1845), which showed CED3 -independent DNase activity (Figure 2B, lanes 7 and 8), bound DNA with or without CED-3 treatment (Figure $6 \mathrm{~A}$, lanes 7 and 8). Thus, the conversion of DCR-1 from an RNase into a DNase likely results from its acquired DNA-binding ability after CED-3 cleavage.

As the one half RNase IIIa domain in tDCR-1 is necessary for DNase activity (Figure 5C, lanes 5 and 6), we tested whether it is important for DNA binding. When the two remaining catalytic residues in the one half RNase IIIa were mutated (D1510A/E1513A), tDCR-1 lost its ability to bind DNA (Figure 6B, lanes 5 and 6). This result indicates that the one half RNase IIIa domain is indispensable for both DNA-binding and DNase activities and may cooperate with the intact RNase IIIb domain to bind and process the DNA substrate.

New DNases can be engineered using different RNase III domains

As the four catalytic acidic residues in the RNase III family are highly conserved $[9,10]$ and are critical for 
tDCR-1 DNase activity [11], we investigated whether other RNase III domains might substitute for DCR1 RNase III domains to generate a similar DNase. We first tested whether we could build an alternative version of tDCR-1 using a complete RNase IIIa domain and a partial RNase IIIb domain. The resulting protein, DCR-1(1297-1670), displayed both DNA-binding and DNase activities similar to tDCR-1 (Figure 7A and 7B, lanes 1-4 and Supplementary information, Figure S1F, lane 3, and Figure S4). Importantly, DCR-1(1297-1670) with a D1472E substitution that blocks in vivo cleavage by CED-3 to preserve an intact RNase IIIa domain completely rescued the cell death defect of $d c r-1(o k 247)$ animals (Figure 7C), indicating that it is a functional equivalent of tDCR-1.

We then replaced the majority of the DCR-1 RNase IIIb domain (residues 1611-1740) with the homologous sequence from $E$. coli RNase III (bRNaseIII; residues 38-128) (Figure 7A). Remarkably, the resulting hybrid protein, DCR-1(1297-bRNaseIII-1845), displayed a CED-3-activated DNase activity indistinguishable from that of DCR-1 or DCR-1(1297-1845) both in vitro and in vivo (Figure 7B, lanes 5-8 and 7D), despite the fact that the new tDCR-1 hybrid engineered is $5 \mathrm{KD}$ smaller than tDCR-1 (Supplementary information, Figure S1F, lanes 4-7). This result suggests that very distantly related RNase III enzymes have an intrinsic ability to cleave DNA and can switch their substrate specificity if they are post-translationally modified, such as proteolytic cleavage, or paired with an appropriate partner that enables them to bind DNA.

\section{Discussion}

In this study, we used a combination of in vitro bio- chemical assays and in vivo functional analyses to probe the mechanistic basis of caspase-mediated conversion of DCR-1 ribonuclease to a DNase, which initiates apoptotic chromosome fragmentation in C. elegans. Our in vitro biochemical analyses demonstrate that various DCR1 protein sequences attached N-terminally to tDCR-1 inhibited both its DNA-binding and DNase activities. Consistently, only DCR-1 variants that exhibited DNAbinding and DNase activities in vitro could promote apoptosis in C. elegans. Our study thus suggests a strong correlation between caspase-mediated activation of DNA binding and activation of DNase activity of the DCR-1 ribonuclease, which underlies this unexpected RNase to DNase conversion.

How then could CED-3 cleavage of DCR-1 activate a DNA-binding activity? The intramolecular dimer formed by the two RNase III domains in the dicer ribonucleases has been proposed to process dsRNA substrates, with each RNase III domain hydrolyzing one RNA strand through a catalytic center composed of eight highly conserved acidic residues $[12,13]$. Cleavage of DCR-1 in the middle of the RNase IIIa domain by CED-3 would disrupt the formation of this stable intramolecular dimer and results in loss of the dsRNA-dicing activity of DCR1 as we have demonstrated previously [11]. Interestingly, DCR-1(1297-1845) and DCR-1(1345-1845), both of which retain all catalytic residues, display greatly impaired dsRNA-dicing activity and generate inaccurate dicing products (Supplementary information, Figure S5A, lanes 2, 4 and 6), likely due to loss of the PAZ domain that determines the specific length of dsRNA cleavage by dicer $[9,10,18,19]$. By contrast, DCR1(1420-1845) and DCR-1(1473-1845), both of which lose two of the four acidic catalytic residues in RNase IIIa, show no ability to cleave dsRNA (Supplementary
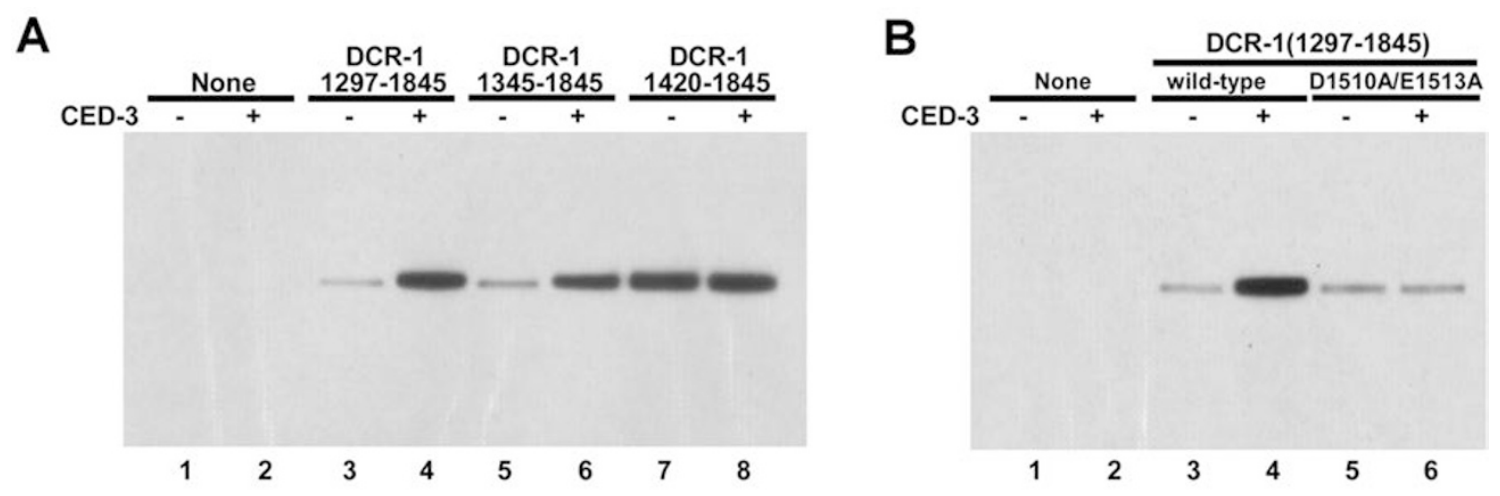

Figure 6 DNA-binding assays with various DCR-1 mutants. (A) Inhibition of the DNA-binding ability of tDCR-1 by the sequences from DCR-1(1297-1472) and DCR-1(1345-1472), but not by DCR-1(1420-1472). (B) tDCR-1 DNA binding was abolished by substitutions of the two catalytic residues in the RNase IIla domain. 
A

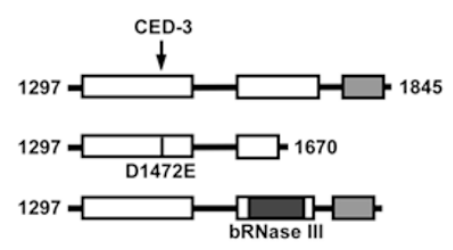

B

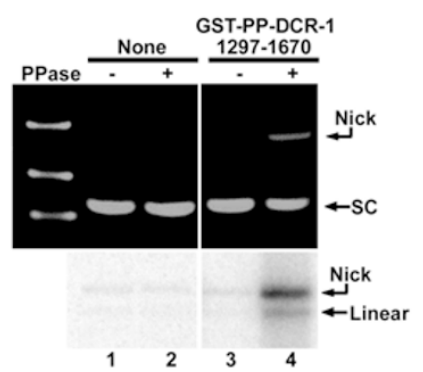

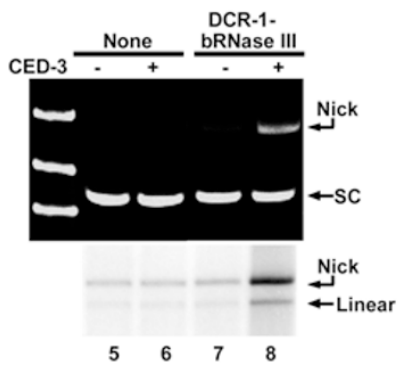

C

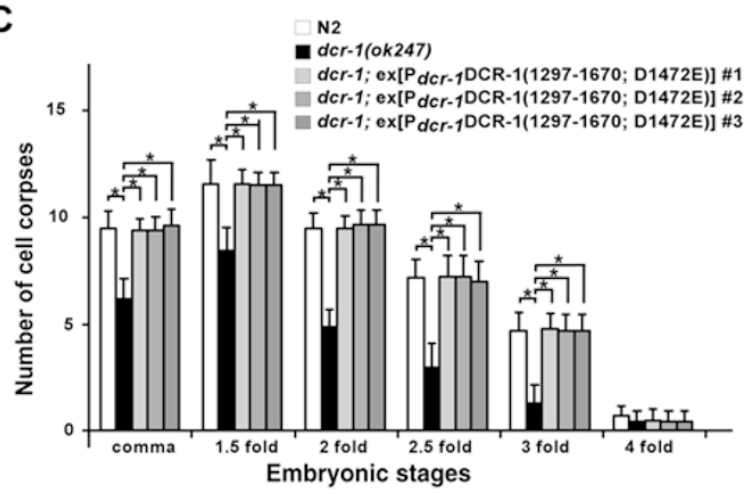

D

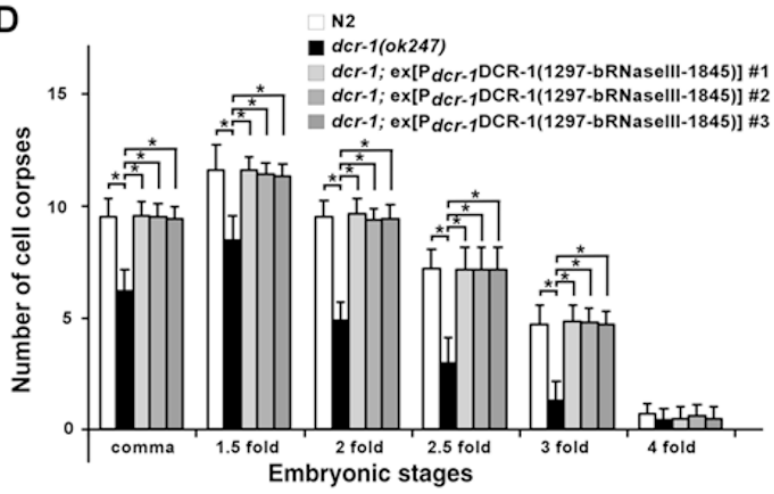

Figure 7 Creation of new DNases using other RNase III nuclease or domains. (A) A schematic diagram of new DNases (see Materials and Methods). The arrow indicates CED-3 cleavage site at Asp1472. (B) DCR-1(1297-1670) shows a similar DNase activity to tDCR-1 and a bacterial RNase III-DCR-1 hybrid also shows a similar CED-3-activated DNase activity. (C, D) Rescue of the cell death defect of $d c r-1$ (ok247) animals by new DNases. Cell corpses were scored in N2, dcr-1(ok247), and dcr-1(ok247) animals carrying the following transgenes: (C) ex[ $\left.P_{d c r-1} D C R-1(1297-1670 ; D 1472 E)\right]$ arrays 1 to 3, and (D) ex[P $P_{d c r-1} D C R-1(1297-b R N a s-$ elll-1845)] arrays 1 to 3. The $x$ axis represents different embryonic stages, $y$ axis represents average number of cell corpses scored and error bars represent standard deviations (SD). ${ }^{*} P<0.001$. All other points had $P>0.05$.

information, Figure S5A, lanes 8 and 10). In the cases of DCR-1(1297-1845) and DCR-1(1345-1845), CED-3 cleavage results in complete loss of their residual RNase activity and gain of a new DNase activity (Figure 1B and Supplementary information, Figure S5, lanes 5 and 7), providing further evidence for CED-3-dependent conversion of DCR-1 RNase to DNase. In vivo, these DCR-1 truncations fail to rescue the abnormal vulva phenotypes (bursting vulva and protruding vulva) of $d c r-1$ (ok247) animals (Supplementary information, Table S1), which are due to a defect in appropriate biogenesis of miRNAs [3].

Moreover, the one half RNase IIIa domain generated by CED-3 cleavage could become more flexible, leading to the formation of a loose intramolecular dimer that instead binds and processes DNA. Indeed, when we performed molecular modeling to simulate the structures of C. elegans DCR-1 before and after CED-3 cleavage (see Materials and Methods), a major conformation shift was suggested. In the before-cleavage model, the two RNase III domains of DCR-1 form an intramolecular dimer similar to that of Giardia Dicer (Supplementary information, Figure S6A) [13] and the homodimer formed by human Dicer RNase IIIb domains [20]. The two catalytic sites, each of which consists of four acidic residues, are exposed on the surface of a shallow groove and are $\sim 20$ $\AA$ apart, which is similar to the distance between catalytic sites of RNase III members and is optimal for hydrolysis of one RNA strand of dsRNA by each RNase III domain (Figure 8A) [12, 13, 21]. Cleavage of the RNase IIIa domain in the middle by CED-3, as predicted by the post-cleavage model, appears to result in the rotation of the remaining RNase IIIa domain from its original position towards the RNase IIIb domain, generating a deep, narrower groove with $\sim 21 \AA$ cleft width (Figure 8B), while the core structure of the RNase IIIb domain seems to have no significant changes (Supplementary information, Figure S6B). This conformational change of the one half RNase IIIa domain could allow tDCR-1 to cradle the DNA substrate, which is predominantly in B-form in cells with a diameter around $20 \AA$ [22]. As a result, DCR1 is converted into a nuclease that binds and processes 
DNA, which has been confirmed by our DNA-binding and nuclease assays (Figures 1, 6, and Supplementary information, Figure S5).

Most of our biochemical observations are consistent with the simulated DCR-1 and tDCR-1 models. For example, addition of an extra protein sequence N-terminal to tDCR-1 may prevent the formation of the DNA-binding cleft between the one half RNase IIIa and RNase IIIb, leading to inhibition of tDCR-1 DNA-binding and DNase activities. This is observed with DCR-1(1297-1845) and DCR-1(1345-1845), both of which are incapable of binding and cleaving DNA without CED-3, but not with DCR-1(1405-1845) or DCR-1(1420-1845), which has a shorter $\mathrm{N}$-terminal sequence and can bind and cleave DNA independent of CED-3 (Figures 1B, 2B and 6A). In the before-cleavage model, residues 1297-1404, which are predicted to form several $\alpha$-helices lying between the one half RNase IIIa domain and the RNase IIIb domain, may prevent the conformational change of the one half RNase IIIa domain and formation of the DNA-binding cleft or block access to the DNA-binding cleft (Figure 8C). On the other hand, residues 1405-1472 appear to situate outside the interface between the one half RNase IIIa domain and the RNase IIIb domain, and thus, do not interfere with DNA binding or affect the DNase activity of tDCR-1 (Figure 8C). In particular, residues 13851404 appear to form an $\alpha$-helix that stretches towards the RNase IIIa/RNase IIIb interface of tDCR-1, interfering with the access to the DNA-binding cleft of tDCR-1 (Figure 8C). Therefore, compared with DCR1(1405-1845), which displays a CED-3-independent DNase activity, the extra 20 residues in DCR-1(13851845 ) render it an inactive DNase before CED-3 cleavage. The observations that an unrelated $\alpha$-helix replacement of residues 1385-1404 in DCR-1(1385-1845) keeps it as a CED-3-dependent DNase, but the flexible GGGSrepeat replacement turns it into a CED-3-independent DNase provide support for the validity of the simulated models. The findings that unrelated proteins with rigid structures, such as GFP and GST [23, 24], potently inhibit tDCR-1 DNase activity when fused to its N-terminus also provide further support to the simulated models.

In our biochemical assays, mutations of the two remaining catalytic residues in the one half RNase IIIa domain (D1510A/E1513A) result in loss of tDCR-1 DNAbinding and DNase activities (Figure 5C and Figure 6B). Consistently, our simulated tDCR-1 model suggests that these two residues are positioned on the surface of the DNA-binding cleft formed by the one half RNase IIIa and the intact RNase IIIb (Figure 8B) and mutations of these two residues likely prevent the interaction of the one half RNase IIIa with DNA. Moreover, as loss of two catalytic acidic residues in the RNase IIIb domain greatly reduces hydrolysis of DNA by tDCR-1 and hydrolysis of dsRNA by DCR-1 [11], the one half RNase IIIa domain with only two catalytic acidic residues probably is inefficient or incapable of cleaving DNA, leading to cleavage of the DNA substrate on one strand by the intact RNase IIIb domain. This is indeed observed with tDCR1 , which generates mostly nicked open circular DNA by nicking one strand of the supercoiled substrate (Figure 1B, lane 4).

Lastly, our in vitro and in vivo results and the simulated model also suggest that new DNases can be generated by pairing an intact RNase III domain with a more flexible partial RNase III domain or a domain that helps cramp DNA to the intact RNase III domain. Indeed, a reverse tDCR-1 with a complete RNase IIIa and a partial RNase IIIb or a hybrid tDCR-1 in which the majority of RNase IIIb domain is replaced by bacterial RNase III functions as a DNase in vitro, and remarkably, fully substitutes for tDCR-1 to promote apoptosis in vivo. Comprehensive structural analyses, in particular, the determination of DCR-1 and tDCR-1 structures with or without nucleic acid substrates, will be critical for elucidating the structural and mechanistic detail of DCR-1 RNase to DNase conversion and conversion of a ribonuclease to a DNase in general.

Our biochemical and functional analyses of tDCR-1 reveal a previously unreported mechanism by which an RNase can be converted into a DNase. Our study also suggests that RNase III enzymes, and potentially other RNases using a similar metal-ion-mediated catalytic mechanism, have the intrinsic capability to cleave DNA and can be converted into a DNase through appropriate post-translational processing or pairing with a proper partner that enables it to hold on to a DNA substrate. Given the vital roles of RNA and DNA in regulating all aspects of cellular functions, similar mechanisms could be used to mediate substrate switch of other ribonucleases, DNases, RNA- or DNA-binding proteins, thereby increasing the ability of nucleic acid-binding proteins or enzymes to regulate diverse biological processes or to promote rapid and drastic changes in cellular events, such as the switch from life to death of a cell catalyzed by DCR-1.

\section{Materials and Methods}

\section{Strains and transgenic animals \\ C. elegans strains were maintained using standard procedures [25]. Transgenic animals were generated as described previously [26]. The DCR-1 constructs were injected into $d c r-1$ (ok247)/hT2 animals at $20 \mu \mathrm{g} / \mathrm{ml}$ along with the pTG96 plasmid (at $20 \mu \mathrm{g} / \mathrm{ml}$ ) as a co-injection marker. The pTG96 plasmid contains a sur-5::gfp}


A

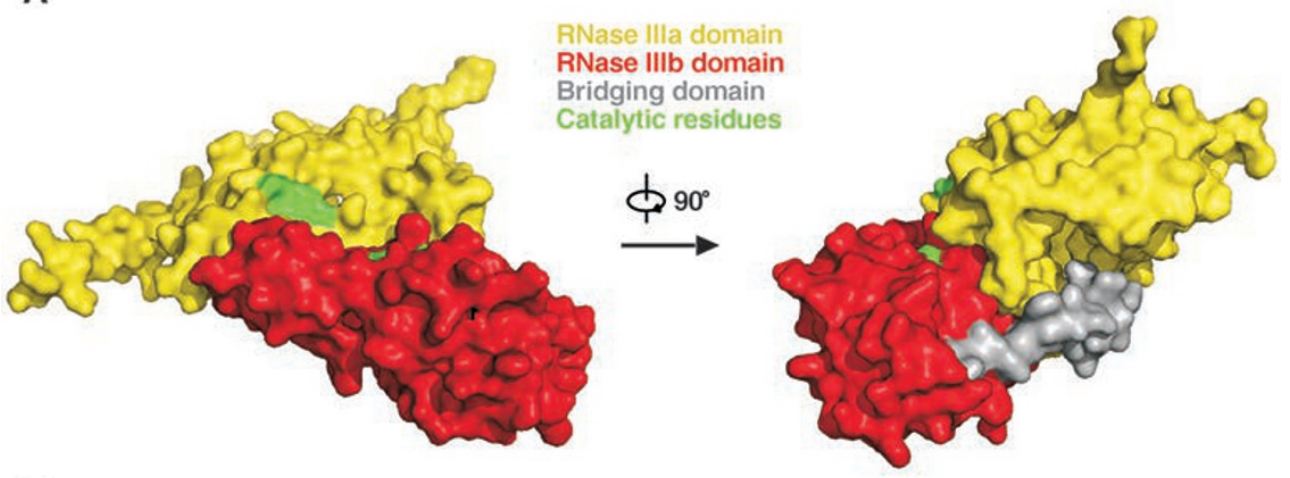

$\mathbf{B}$
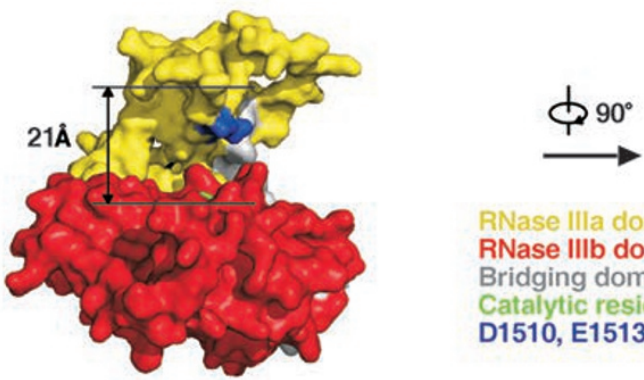

RNase Illa domain RNase IIIb domain Bridging domain Catalytic residues D1510, E1513
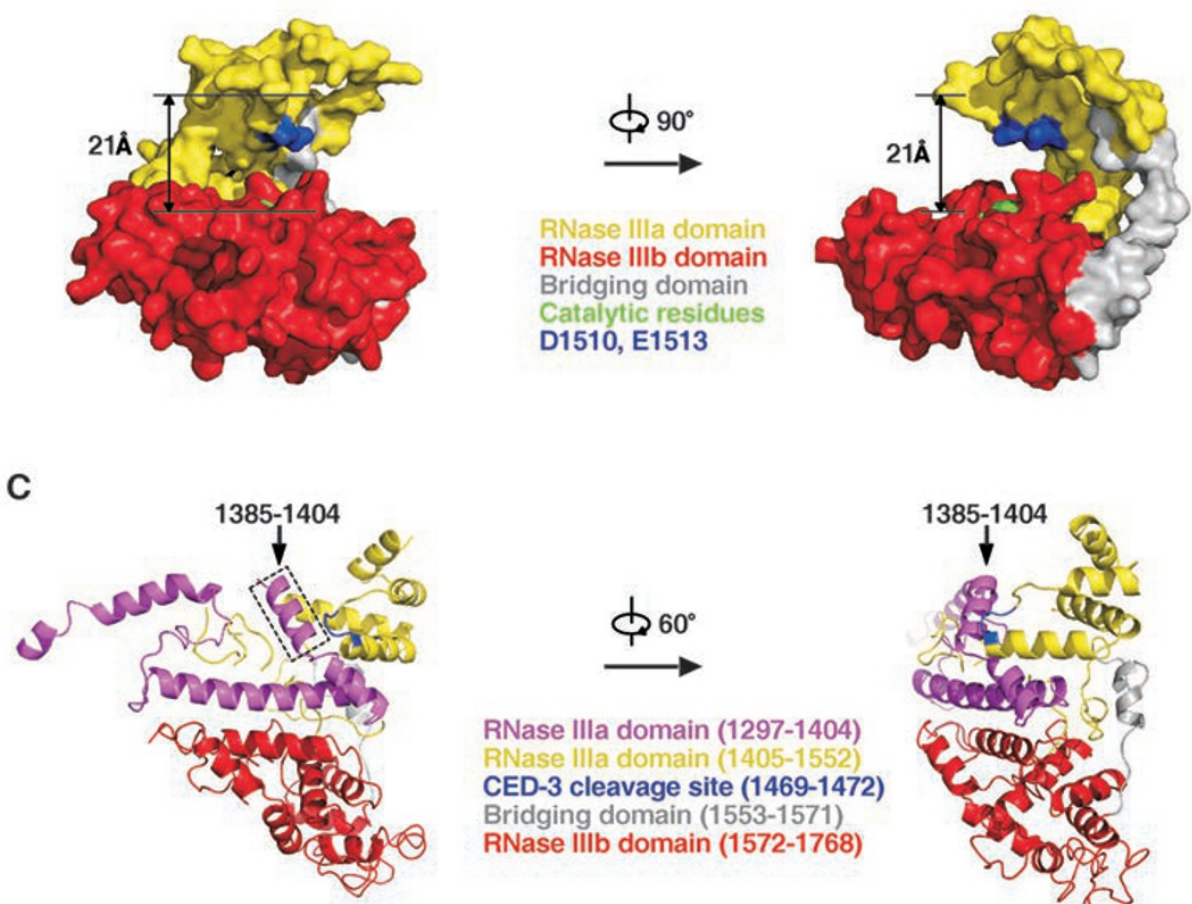

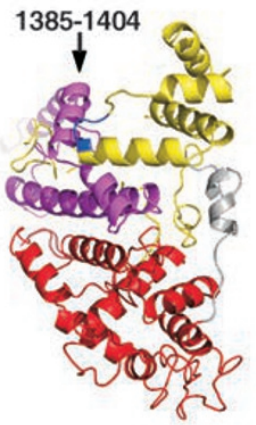

Figure 8 Structural modeling of DCR-1 before and after CED-3 cleavage. (A) The modeled structure of DCR-1(1297-1768) with two complete RNase III domains, which are shown in yellow and red, respectively, and the bridging domain shown in grey. Catalytic residues are shown in green. Two perpendicular views are presented. The two catalytic sites lie on a flat surface, which can be easily accessed by the dsRNA substrate. (B) The modeled structure of DCR-1(1473-1768) with one and a half RNase III domains. The color labeling of RNase III domains and catalytic residues is as presented in A, except that the two catalytic residues in the one half RNase Illa domain are shown in blue instead. After CED-3 cleavage, the one half RNase IIla domain becomes flexible and rotates perpendicularly relative to the RNase IIIb domain to form a deep cavity with a width of $\sim 21 \AA$, which can accommodate a DNA substrate. (C) In the ribbon diagram of the before-cleavage model, residues 1297-1404 (in magenta) form several $\alpha$-helices positioned between the RNase IIIb domain and the one half RNase IIla domain. The $\alpha$-helix consisting of residues $1385-1404$ is indicated by a dashed box. The CED-3 recognition and cleavage site STTD (residues 1469-1472) is shown in blue.

translational fusion that is expressed in all somatic cells in most developmental stages [27].

\section{Quantification of cell corpses}

The number of cell corpses in living embryos was scored using Nomarski optics as described previously [28]. Stages of embryos examined were: comma, 1.5-fold, 2-fold, 2.5-fold, 3-fold, and 4-fold. 15 embryos were counted for each developmental stage. The significance of differences between different genetic backgrounds was determined by two-way ANOVA, followed by 
the Bonferroni comparison.

\section{Molecular biology}

To make truncated tDCR-1 clones, the boundaries of the helicase domain (1-500), the PAZ domain (740-950), the RNase IIIa domain (1297-1552), the RNase IIIb domain (1572-1768) and dsRNA-binding domain (1769-1845) are defined according to published sequence alignment $[20,29,30]$. Full-length $d c r-1$ cDNA and genomic clones were obtained as described previously [11]. To construct truncated pET-41b-DCR-1 plasmids, dcr-1 cDNA fragments encoding residues 1297-1845, 1345-1845, $1385-1845,1405-1845,1420-1845,1473-1845,1560-1845$ and 1297-1670 were amplified from full-length $d c r-1$ cDNA using PCR and subcloned into the pET-41b vector through its SpeI and NotI sites, with a PPase cleavage site LEVLFQGP inserted between the GST-tag and truncated DCR-1 proteins. To make pET41b-DCR-1(D1510A/E1513A) or D1472E-containing mutants, a two-step PCR was designed to generate the indicated mutations. To make pET-41b-DCR-1(1297-bRNaseIII-1845), the DNA sequence encoding residues 1611-1740 of DCR-1 was replaced by a sequence encoding residues $38-128$ of $E$. coli RNase III. To make the constructs replacing DCR-1 residues 1385-1404, DNA fragments encoding DQWGVELGKQLAKKIEPELDG, an $\alpha$-helix from rabbit phosphoglucose isomerase (PDB code: 1DQR) retrieved from the Linker Database (http:/www.ibi.vu.nl/programs/ linkerdbwww/) [31] or encoding a sequence with unstructured repeats SGGGSGGGSGGGSGGGSGGGS were synthesized and inserted before the cDNA clone encoding DCR-1(1405-1845). To make pET-41b-GFP-DCR-1(1473-1845) and pET-41b-GFPDCR-1(1469-1845) plasmids, the cDNA fragment encoding GFP was amplified and fused to the N-terminus of DCR-1(1473-1845) or DCR-1(1469-1845). To generate pET-41b-DCR-1(1-1845; $\Delta 1385-1404)$ and pET-41b-DCR-1(1-1384-GS-1405-1845), the sequence encoding residues 1385-1404 of DCR-1 was removed or replaced with the GGGS-repeats through a two-step PCR subcloning method. To express GST-DCR-1 fusion proteins in insect cells, GST coding region with a PPase cleavage site was amplified from pGEX-6P1 (GE Healthcare) and inserted into the pFastBac1 (Invitrogen) vector to facilitate the construction of GST fusion clones. Various $d c r-1$ cDNA fragments with a C-terminal six Histidine (His6) and FLAG tag were inserted via BamHI/NotI sites. The resulting pFastBac1 constructs were then transformed into DH10Bac to produce recombinant baculovirus for insect cell transfection according to the manufacturer's instructions (Invitrogen).

For in vivo rescue experiments, the $\mathrm{P}_{d c r-1}$ DCR-1 plasmid was used as the backbone to make constructs that express various DCR1 mutants under the control of the endogenous $d c r-1$ promoter, as previously described [11]. Specifically, $\mathrm{P}_{d c r-1}$ DCR-1 was used as a DNA template to remove the genomic regions encoding residues $1-739,1-1296,1-1344,1-1384,1-1404,1-1419$, and 1-1559 or to generate mutations in DCR-1 (D1472E or D1510A/E1513A), using the Quick Change mutagenesis kit (Stratagene). To make the in vivo constructs replacing DCR-1 residues 1385-1404, DNA fragments encoding the helix DQWGVELGKQLAKKIEPELDG, or unstructured repeats SGGGSGGGSGGGSGGGSGGGS were synthesized and inserted right after the start codon of $\mathrm{P}_{d c r-1}$ DCR-1(1405-1845) and $\mathrm{P}_{d c r-1}$ DCR-1(1405-1845; D1472E). To construct $\mathrm{P}_{d c r-1} \mathrm{GFP}-$ DCR-1(1469-1845) or P ${ }_{d r-1}$ GFP-DCR-1(1473-1845), the DNA sequence encoding DCR-1 residues 1-1468 or 1-1472 was replaced with the GFP coding sequence. To construct $\mathrm{P}_{d c r}$ ${ }_{1}$ DCR-1(1297-1670; D1472E), the DNA sequence encoding residues 1671-1845 was removed from the $\mathrm{P}_{d c r-1}$ DCR-1(1297-1845; D1472E) plasmid. To construct $\mathrm{P}_{d c r-1}$ DCR-1(1297-bRNaseIII-1 845), the $\mathrm{P}_{d c r-1}$ DCR-1(1297-1845) plasmid was used as a template and the DNA sequence encoding residues 1611-1740 was replaced with a sequence encoding residues 38-128 of E. coli RNase III. All clones were confirmed by DNA sequencing.

\section{Protein expression and purification}

All GST-DCR-1 fusion proteins used in DNase and RNase assays were synthesized using the TNT Coupled Reticulocyte Lysate System (Promega) and affinity purified by Glutathione Sepharose 4B beads (GE Healthcare) as described in detail previously [11]. To obtain GST-DCR-1 fusion proteins used in DNA-binding experiments, Sf9 cells were infected with recombinant baculovirus and harvested after $60 \mathrm{~h}$. The cells were lysed and recombinant proteins were purified sequentially by $\mathrm{Ni}^{2+}$ Sepharose resin (GE Healthcare) and Glutathione Sepharose 4B resin. Purified GST-DCR-1 fusion proteins were then cleaved using the PreScission Protease at room temperature for $2 \mathrm{~h}$ to remove the GST tag.

\section{Plasmid cleavage assay and TdT assay}

For the DNase activity assay, purified DCR-1 proteins were incubated with or without CED-3 at $30^{\circ} \mathrm{C}$ for $1 \mathrm{~h}$ and then with 200 ng of supercoiled pPD49.78 plasmid DNA in the DNase buffer (20 $\mathrm{mM}$ HEPES-KOH, $10 \mathrm{mM} \mathrm{KCl}, 3 \mathrm{mM} \mathrm{MgCl}, 0.5 \mathrm{mM} \mathrm{DTT}$ ) at $37^{\circ} \mathrm{C}$ for another $1 \mathrm{~h}$. Half of the DNA samples were resolved on $1 \%$ agarose gel, stained with GelRed (Biotium), and photographed under UV light. Tans2K plus DNA (Transgene) was used as size markers. The other half was labeled as with Terminal Deoxynucleotidyl Transferase (TdT) in a TdT buffer (20 mM Tris-acetate, 50 $\mathrm{mM}$ potassium acetate, $10 \mathrm{mM}$ magnesium acetate, $250 \mu \mathrm{M}$ cobalt chloride, $\left.\alpha-{ }^{32} \mathrm{P}-\mathrm{dCTP}\right)$ at $37{ }^{\circ} \mathrm{C}$ for $2 \mathrm{~h}$. The samples were then passed through G-50 MicroSpin columns (GE Healthcare) and resolved on $1 \%$ agarose gel. The gel was fixed with $10 \%$ methanol $/ 10 \%$ acetic acid and dried on a SpeedGel gel drier (Thermo) before autoradiography.

\section{DNA-binding assay}

DCR-1 proteins with a C-terminal FLAG tag purified from insect cells were incubated with anti-FLAG M2 beads (SigmaAldrich) at $4{ }^{\circ} \mathrm{C}$ for $1 \mathrm{~h}$, washed with DNA-binding buffer (20 mM HEPES-KOH, $10 \mathrm{mM} \mathrm{KCl,} 3 \mathrm{mM} \mathrm{MgCl}_{2}$ ), and then incubated with or without CED-3 at $30{ }^{\circ} \mathrm{C}$ for another $1 \mathrm{~h}$. One $\mu \mathrm{g}$ of pPD49.78 plasmid DNA was added to the mixture, incubated at $37^{\circ} \mathrm{C}$ for $20 \mathrm{~min}$ and then irradiated with $254 \mathrm{~nm} \mathrm{UV}$ for 20 min on ice. The beads were then washed three times with the binding buffer. Bound DNA was digested by BamHI, labeled with biotinylated dATP by Klenow (New England Biolabs), and resolved on agarose gel. The samples were then transferred onto Nylon+ membrane (Millipore) and biotin-labeled DNA was detected using HRP-conjugated streptavidin (Thermo).

\section{Ribonuclease assay}

The DCR-1 ribonuclease assay was performed as described previously [11]. Briefly, ${ }^{32} \mathrm{P}-1 \mathrm{abeled} 189 \mathrm{bp}$ dsRNA was synthesized by T7 High Yield RNA Synthesis Kit (New England 
Biolabs) using pPD129.36 as the template and purified by G-50 MicroSpin columns (GE Healthcare). GST-DCR-1 proteins were synthesized using the TNT Coupled Reticulocyte Lysate System and affinity purified by Glutathione Sepharose 4B beads. After PPase cleavage, DCR-1 proteins were incubated with ${ }^{32} \mathrm{P}$-labeled 189 bp dsRNA in a buffer containing $20 \mathrm{mM}$ HEPES-KOH, 10 $\mathrm{mM} \mathrm{KCl}, 3 \mathrm{mM} \mathrm{MgCl}$, and $0.5 \mathrm{mM} \mathrm{DTT}$ at $37^{\circ} \mathrm{C}$ for $2 \mathrm{~h}$ in the absence or presence of CED-3. The samples were then resolved on a $20 \%$ native polyacrylamide gel. The gel was first stained with GelRed to visualize siRNA marker (New England Biolabs) and then fixed and dried for autoradiography.

\section{CED-3 protease assay}

CED-3 protease assays were carried out as described previously [32]. Briefly, GST-DCR-1 proteins were synthesized and labeled with ${ }^{35} \mathrm{~S}$-methionine using the TNT Coupled Reticulocyte Lysate System (Promega) and incubated with purified CED-3 at $30{ }^{\circ} \mathrm{C}$ for $1 \mathrm{~h}$. The reactions were then resolved by $15 \%$ SDS-PAGE.

\section{Structural modeling}

The three-dimension structures of DCR-1 before and after CED-3 cleavage were simulated using $a b$ initio and homology modeling methods. In the ab initio method, the before-cleavage DCR-1 sequence from amino acid 1297 to 1768 was used as an input to obtain the fragment structure templates. The three-residue fragments and nine-residue fragments were generated from the ROBETTA fragment server (http://robetta.bakerlab.org/fragmentsubmit.jsp). The ab initio modeling protocol from Rosetta 3.3 [33] was implemented to take advantage of the above fragment information to generate 50000 models. The model with a high conformational energy score and in agreement with the structures of Giardia dicer and other RNase III dimers was selected as the final candidate model. Briefly, the four catalytic residues in each RNase III domain should be adjacent to one another to constitute a catalytic site, and the two catalytic sites should be oriented to the same direction on the surface of the intramolecular dimer composed of the two RNase III domains, with a distance agreeing with the width of the major groove of dsRNA.

The structure of the after-cleavage DCR-1 protein (amino acid 1473-1768) was predicted using the homology modeling methods JACKAL [34] and I-TASSER [35], with totally 200 models generated. Because tDCR-1's DNase activity is dependent on the presence of four catalytic residues in RNase IIIb domain and $\mathrm{Mg}^{2+}$ [11], the integrity of the catalytic site in RNase IIIb domain should be preserved. Among top five predicted models with highest conformational energy scores, only one model met this criterion and was selected as the final model (Figure 8B).

\section{Acknowledgments}

We thank T Blumenthal, N Pace, and M Yarus (University of Colorado) for comments and members of the Xue lab for helpful discussions. Computational resources used in this study were supported by Tsinghua National Laboratory for Information Science and Technology. This work was supported by a Tsinghua-Peking University Life Science Center fund, the 973 Program 2013CB945602, and NIH grants R01 GM59083 and R01 GM79097.

\section{References}

1 Hammond SM, Bernstein E, Beach D, Hannon GJ. An RNAdirected nuclease mediates post-transcriptional gene silencing in Drosophila cells. Nature 2000; 404:293-296.

2 Bernstein E, Caudy AA, Hammond SM, Hannon GJ. Role for a bidentate ribonuclease in the initiation step of RNA interference. Nature 2001; 409:363-366.

3 Grishok A, Pasquinelli AE, Conte D, et al. Genes and mechanisms related to RNA interference regulate expression of the small temporal RNAs that control C. elegans developmental timing. Cell 2001; 106:23-34.

4 Hutvagner G, McLachlan J, Pasquinelli AE, Balint E, Tuschl $\mathrm{T}$, Zamore PD. A cellular function for the RNA-interference enzyme dicer in the maturation of the let-7 small temporal RNA. Science 2001; 293:834-838.

5 Knight SW, Bass BL. A role for the RNase III enzyme DCR-1 in RNA interference and germ line development in Caenorhabditis elegans. Science 2001; 293:2269-2271.

6 Pham JW, Pellino JL, Lee YS, Carthew RW, Sontheimer EJ. A dicer-2-dependent 80 s complex cleaves targeted mRNAs during RNAi in Drosophila. Cell 2004; 117:83-94.

7 Lee YS, Nakahara K, Pham JW, et al. Distinct roles for Drosophila dicer-1 and dicer-2 in the siRNA/miRNA silencing pathways. Cell 2004; 117:69-81.

8 Duchaine TF, Wohlschlegel JA, Kennedy S, et al. Functional proteomics reveals the biochemical niche of $C$. elegans DCR-1 in multiple small-RNA-mediated pathways. Cell 2006; 124:343-354.

9 MacRae IJ, Doudna JA. Ribonuclease revisited: structural insights into ribonuclease III family enzymes. Curr Opin Struct Biol 2007; 17:138-145.

10 Jaskiewicz L, Filipowicz W. Role of dicer in posttranscriptional RNA silencing. Curr Top Microbiol Immunol 2008; 320:7797.

11 Nakagawa A, Shi Y, Kage-Nakadai E, Mitani S, Xue D. Caspase-dependent conversion of dicer ribonuclease into a death-promoting deoxyribonuclease. Science 2010; 328:327334.

12 Zhang H, Kolb FA, Jaskiewicz L, Westhof E, Filipowicz W. Single processing center models for human dicer and bacterial RNase III. Cell 2004; 118:57-68.

13 MacRae IJ, Zhou K, Li F, et al. Structural basis for doublestranded RNA processing by dicer. Science 2006; 311:195198.

14 Parrish JZ, Xue D. Functional genomic analysis of apoptotic DNA degradation in C. elegans. Mol Cell 2003; 11:987-996.

15 Parrish JZ, Xue D. Cuts can kill: the roles of apoptotic nucleases in cell death and animal development. Chromosoma 2006; 115:89-97.

16 Cole C, Barber JD, Barton GJ. The Jpred 3 secondary structure prediction server. Nucleic Acids Res 2008; 36:197201.

17 Yang W. Nucleases: diversity of structure, function and mechanism. Q Rev Biophys 2011; 44:1-93.

18 Ma E, Zhou K, Kidwell MA, Doudna JA. Coordinated activities of human dicer domains in regulatory RNA processing. J Mol Biol 2012; 422:466-476.

19 MacRae IJ, Zhou K, Doudna JA. Structural determinants of 
RNA recognition and cleavage by dicer. Nat Struct Mol Biol 2007; 14:934-940.

20 Takeshita D, Zenno S, Lee WC, Nagata K, Saigo K, Tanokura M. Homodimeric structure and double-stranded RNA cleavage activity of the C-terminal RNase III domain of human dicer. J Mol Biol 2007; 374:106-120.

21 Du Z, Lee JK, Tjhen R, Stroud RM, James TL. Structural and biochemical insights into the dicing mechanism of mouse dicer: a conserved lysine is critical for dsRNA cleavage. Proc Natl Acad Sci USA 2008; 105:2391-2396.

22 Lehninger AL, Nelson DL, Cox MM, eds. Lehninger principles of biochemistry. 4th Edition. New York: W.H. Freeman, 2005.

23 Ormo M, Cubitt AB, Kallio K, Gross LA, Tsien RY, Remington SJ. Crystal structure of the Aequorea victoria green fluorescent protein. Science 1996; 273:1392-1395.

24 McTigue MA, Williams DR, Tainer JA. Crystal structures of a schistosomal drug and vaccine target: glutathione S-transferase from Schistosoma japonica and its complex with the leading antischistosomal drug praziquantel. $J \mathrm{Mol}$ Biol 1995; 246:21-27.

25 Brenner S. The genetics of Caenorhabditis elegans. Genetics 1974; 77:71-94.

26 Mello CC, Kramer JM, Stinchcomb D, Ambros V. Efficient gene transfer in C.elegans: extrachromosomal maintenance and integration of transforming sequences. EMBO J 1991; 10:3959-3970.
27 Gu T, Orita S, Han M. Caenorhabditis elegans SUR-5, a novel but conserved protein, negatively regulates LET-60 Ras activity during vulval induction. Mol Cell Biol 1998; 18:45564564.

28 Parrish J, Li L, Klotz K, Ledwich D, Wang X, Xue D. Mitochondrial endonuclease $\mathrm{G}$ is important for apoptosis in $C$. elegans. Nature 2001; 412:90-94.

29 Provost P, Dishart D, Doucet J, Frendewey D, Samuelsson $\mathrm{B}$, Radmark O. Ribonuclease activity and RNA binding of recombinant human Dicer. EMBO J 2002; 21:5864-5874.

30 Krautz-Peterson G, Skelly PJ. Schistosoma mansoni: the dicer gene and its expression. Exp Parasitol 2008; 118:122-128.

31 George RA, Heringa J. An analysis of protein domain linkers: their classification and role in protein folding. Protein Eng 2002; 15:871-879.

32 Xue D, Shaham S, Horvitz HR. The Caenorhabditis elegans cell-death protein CED-3 is a cysteine protease with substrate specificities similar to those of the human CPP32 protease. Genes Dev 1996; 10:1073-1083.

33 Leaver-Fay A, Tyka M, Lewis SM, et al. ROSETTA3: an object-oriented software suite for the simulation and design of macromolecules. Methods Enzymol 2011; 487:545-574.

34 Xiang Z, Honig B. Extending the accuracy limits of prediction for side-chain conformations. J Mol Biol 2001; 311:421-430.

35 Roy A, Kucukural A, Zhang Y. I-TASSER: a unified platform for automated protein structure and function prediction. Nat Protoc 2010; 5:725-738.

(Supplementary information is linked to the online version of the paper on the Cell Research website.) 